\title{
Central melanocortin receptors regulate insulin action
}

\author{
Silvana Obici, Zhaohui Feng, Jianzhen Tan, LiSen Liu, George Karkanias, \\ and Luciano Rossetti
}

Diabetes Research and Training Center, Albert Einstein College of Medicine, Bronx, New York, USA

Address correspondence to: Luciano Rossetti, Albert Einstein College of Medicine, 1300 Morris Park Avenue, Bronx, New York 10461, USA. Phone: (718) 430-4118; Fax: (718) 430-8557; E-mail: rossetti@aecom.yu.edu.

Received for publication April 9, 2001, and accepted in revised form August 8, 2001.

\begin{abstract}
Energy balance and insulin action are tightly coregulated. Leptin regulates energy intake and expenditure partly by modulation of the melanocortin pathway in the hypothalamus. Here we demonstrate potent effects of the melanocortin pathway on insulin action and body distribution of adiposity. Conscious rats received week-long infusions of either a melanocortin receptor agonist, $\alpha$-melanocytestimulating hormone ( $\alpha-\mathrm{MSH})$, or antagonist, SHU9119, in the third cerebral ventricle while food intake was maintained constant in each group. $\alpha-\mathrm{MSH}$ decreased intra-abdominal fat and markedly enhanced the actions of insulin on both glucose uptake and production, while SHU9119 exerted opposite effects. Our findings elucidate a neuroendocrine network that is likely to play a central role in the coupling of energy intake and insulin action.
\end{abstract}

J. Clin. Invest. 108:1079-1085 (2001). DOI:10.1172/JCI200112954.

\section{Introduction}

Insulin resistance is regarded as the main link between obesity and type 2 diabetes mellitus $(1,2)$. While multiple hypotheses have been proposed to explain this association, recent discoveries on the regulation of feeding behavior and energy expenditure (3-6) have refocused attention on the potential role of hypothalamic centers in the regulation of both energy homeostasis and insulin action (Figure 1) (7-13). In fact, leptin has potent effects on glucose tolerance and insulin action, which appear to be independent of its modulation of feeding behavior $(7,9,13)$. Leptin exerts its actions on food intake and weight gain partly by activation of the melanocortin pathway in the hypothalamus and in other areas within the central nervous system (14). The pivotal role of melanocortinergic neurons in the regulation of energy balance is supported by solid genetic $(15,16)$ and pharmacologic (17) evidence.

To examine whether the activity of the melanocortin pathway in the hypothalamus modulates insulin action in vivo we used the natural agonist, $\alpha$-melanocyte-stimulating hormone $(\alpha-\mathrm{MSH})$, and a high-affinity antagonist (SHU9119) of the neural melanocortin receptors type 3 and 4 (MCR3, MCR4) (17) to generate bidirectional variations in the activity of this pathway. Body composition and metabolic parameters were assessed using tracer dilution techniques and pancreatic-insulin clamp studies in conscious rats.

\section{Methods}

Experimental procedures. Thirty-nine male Sprague-Dawley rats (Charles River Laboratories, Wilmington, Massachusetts, USA) were studied (Figure 2). Rats were housed in individual cages and subjected to a standard light-dark
(0600 to 1800 hours/1800 to 0600 hours) cycle. Three weeks before the in vivo study, rats (at $\sim 10$ weeks of age) were equipped with chronic catheters placed in the third cerebral ventricle (18). Rats were anesthetized with intraperitoneal ketamine (Ketaset; $87 \mathrm{mg} / \mathrm{kg}$ ) and xylazine (Rompun; $11 \mathrm{mg} / \mathrm{kg}$ ) and fixed in a stereotaxic apparatus with ear bars and a nose piece set at $+5.0 \mathrm{~mm}$. A 26-gauge stainless steel guide cannula (Plastics One Inc., Roanoke, Virginia, USA) was chronically implanted into the third ventricle using the following coordinates from bregma: anterior-posterior; $+0.2 \mathrm{~mm}$, dorsal-ventral; $-9.0 \mathrm{~mm}$, medial-lateral; 0.0 directly on the midsagittal suture. A 28-gauge dummy cannula was inserted to prevent clogging of the guide cannula. The implant is secured to the skull with Caulk Grip dental cement, and the skin is closed over the implant using wound clips. One week before the study, rats (at $~ 12$ weeks of age) were anesthetized with an intraperitoneal injection of pentobarbital $(50 \mathrm{mg} / \mathrm{kg}$ body weight), and indwelling catheters were inserted in the right internal jugular vein and in the left carotid artery (18-21). The venous catheter was extended to the level of the right atrium, and the arterial catheter was advanced to the level of the aortic arch. All studies were performed in awake, unstressed, chronically catheterized rats. Histological verification of the intracerebroventricular cannula was performed at the end of each experiment using infusion cannulae. Animals were anesthetized and decapitated, and the brains were removed, quick-frozen at $-35^{\circ} \mathrm{C}$ for 2 minutes in isopentane, and stored in tissue matrix at $-76^{\circ} \mathrm{C}$. Frozen brains were mounted and placed in a cryostat and sectioned in the coronal plane. Every fifth $40-\mu \mathrm{m}$ frontal section was examined and the neuroanatomical location of the cannulae tips was verified. 

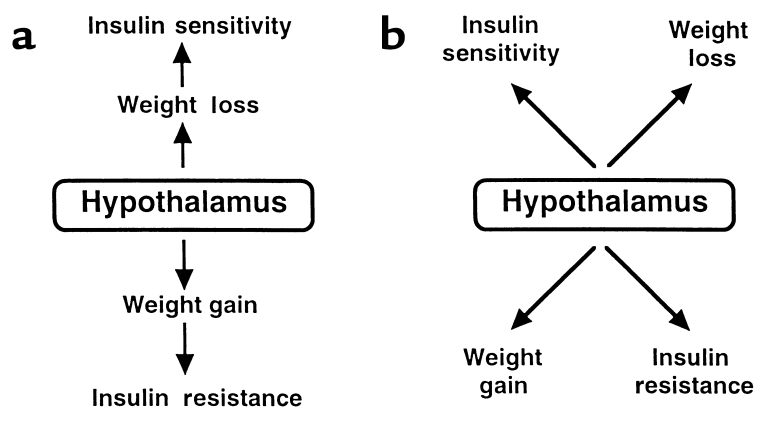

\section{Figure 1}

Hypotheses on the regulation of insulin action by hypothalamic neural pathways. (a) Sequential model: neuronal pathways regulate feeding behavior and energy balance, which in turn modulate insulin action. (b) Parallel model: neuronal pathways concomitantly regulate weight homeostasis and insulin action. The two models are not mutually exclusive.

Implantation of intracerebroventricular osmotic minipumps. One week before the in vivo studies, osmotic minipumps (Alzet Inc., Cupertino, California, USA) were connected to the intracerebroventricular catheter in all rats (Figure 2). The minipump delivered a constant infusion of 0.5 $\mu \mathrm{l} / \mathrm{h}$ for 7 days. Rats were divided in three groups receiving vehicle, $\alpha$-MSH (50 ng/h), or SHU9119 (20 ng/h). The rats receiving SHU9119 were further randomized in rats fed ad libitum and rats pair-fed with the other two groups. Food intake was monitored daily, and the average rate of food intake during intracerebroventricular administration is displayed in Table 1. An additional group of rats received week-long infusion of $\alpha-\mathrm{MSH}$ (50 $\mathrm{ng} / \mathrm{h}$ ) and either an oligodeoxynucleotide (ODN) antisense designed to decrease expression of the MCR4 or a scrambled ODN as a control. Antisense and scrambled ODNs were dissolved in artificial cerebrospinal fluid (CSF) at a concentration of $2 \mathrm{mM}$. The solutions were infused intracerebroventricularly for 7 days using osmotic minipumps at a rate of $1 \mathrm{nmol} / \mathrm{h}$. ODNs were synthesized by Operon Technologies Inc. (Alameda, California, USA). All ODNs contain a phosphothiorate bond between the 1st and 2nd nucleotide and between the 16 th and 17 th nucleotide. A 17-base antisense sequence ( $3^{\prime}$-ACGTCCTTCTACTTGAG-5') complementary to the mRNA of rat MCR4 was chosen from the Genbank database (no. GI1565664). The antisense sequence was scrambled and used to synthesize an ODN for control ( $5^{\prime}$ CCTGAATCATGTCCTTG- $\left.3^{\prime}\right)$. The selected sequence spans the region of mRNA containing the putative AUG starting codon. On the 8th day following initiation of intracerebroventricular infusions, insulin clamp studies were performed in conscious rats fasted for approximately 6 hours, as described previously $(11,19)$. To ensure a similar postabsorptive state, on the night preceding the clamp study all rats received a fixed allotment of chow $(\sim 10 \mathrm{~g})$ that they completely consumed by approximately 0200 hours.

Body composition. Rats received an intra-arterial bolus injection of $20 \mu \mathrm{Ci}$ of tritiated-labeled water $\left({ }^{3} \mathrm{H}_{2} \mathrm{O}\right.$;
New England Nuclear, Boston, Massachusetts, USA), and plasma samples were obtained at 20-minute intervals for 2 hours $(13,18)$. Steady-state conditions for plasma ${ }^{3} \mathrm{H}_{2} \mathrm{O}$-specific activity were achieved within 45 minutes in all studies. Five plasma samples obtained between 1 and 2 hours were used in the calculation of the whole-body distribution space of water. The latter was obtained by dividing the total radioactivity injected (in dpm) by the steady-state specific activity of plasma water (in dpm/ml). Plasma was assumed to be $93 \%$ water. Fat-free mass was calculated as the whole body water distribution space divided by 0.73 and fat mass as the difference of body weight and fat-free mass. Epididymal, perirenal, and omental fat depots were dissected and weighed at the end of each experiment.

Measurements of in vivo glucose kinetics. Briefly, a primed-continuous infusion of HPLC-purified [ $\left.{ }^{3} \mathrm{H}-3\right]$ glucose (New England Nuclear; $40 \mu \mathrm{Ci}$ bolus, 0.4 $\mu \mathrm{Ci} / \mathrm{min}$ ) was administered for the duration of the study $(18,21)$. Two hours after the basal period, primed-continuous infusions of somatostatin (1.8 $\mu \mathrm{g} / \mathrm{kg} / \mathrm{min})$ and regular insulin $(3 \mathrm{mU} / \mathrm{kg} / \mathrm{min})$ were administered, and a variable infusion of a $25 \%$ glucose solution was started at time zero and periodically adjusted to clamp the plasma glucose concentration at approximately $7.2 \mathrm{mM}$ for the rest of the studies. Samples for determination of ${ }^{3} \mathrm{H}$-glucose specific activity were obtained at 10-minute intervals throughout the infusions. The total volume of blood sampled was approximately $5.0 \mathrm{ml} / \mathrm{study}$. To prevent volume depletion and anemia, a solution ( $1: 1 \mathrm{vol} / \mathrm{vol})$ of approxi-

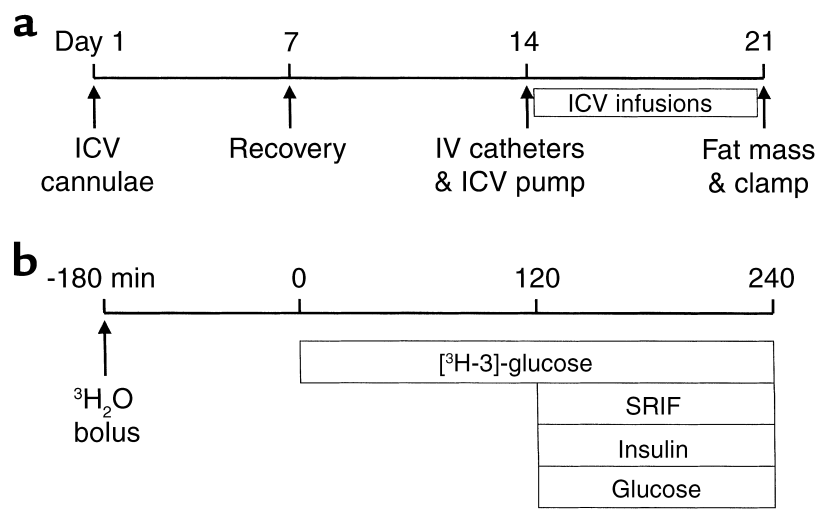

\section{Figure 2}

Schematic representation of the experimental design. (a) Surgical implantation of intracerebroventricular cannulae was performed on day 1 ( $\sim 3$ weeks before the in vivo study). Full recovery of body weight and food intake was achieved by day 7 . Surgical implantation of intravenous catheters and of osmotic minipumps (for intracerebroventricular [ICV] infusions) was performed on day 14. Intracerebroventricular infusions were continued for 7 days. Finally, on day 21, body composition and insulin action were estimated. (b) A bolus of tritiated water was given intravenously 3 hours before starting the tritiated glucose infusion. At $t=0$ a primed-continuous infusion of labeled glucose was initiated and maintained for the remainder of the 4-hour study. Pancreatic-insulin clamp study was initiated at $t=120$ minutes and lasted 120 minutes. 
Table 1

Effect of intracerebroventricular $\alpha-\mathrm{MSH}$ and SHU9119 on food intake and body composition

\begin{tabular}{lcccc}
\hline & Vehicle & $\alpha-M S H$ & SHU & SHU-PF \\
Caloric intake (kcal/d) & $63 \pm 2$ & $62 \pm 4$ & $107 \pm 10^{\mathrm{A}}$ & $65 \pm 6$ \\
BW (g) & $306 \pm 10$ & $291 \pm 5$ & $327 \pm 3^{\mathrm{A}}$ & $292 \pm 9$ \\
$\Delta$ BW (g) & $+4 \pm 3$ & $-22 \pm 6^{\mathrm{A}}$ & $+21 \pm 3^{\mathrm{A}}$ & $-21 \pm 9$ \\
FFM (g) & $265 \pm 11$ & $259 \pm 7$ & $271 \pm 4$ & $249 \pm 11$ \\
FM (g) & $40 \pm 3$ & $32 \pm 6$ & $56 \pm 4^{\mathrm{A}}$ & $43 \pm 7$
\end{tabular}

Osmotic minipumps containing either $\alpha$-MSH $(25 \mu \mathrm{g}$ in $250 \mu \mathrm{l}$ vehicle), SHU9119 (10 $\mu \mathrm{g}$ in $250 \mu$ vehicle), or vehicle were implanted subcutaneously and connected to the third cerebral ventricle (intracerebroventricularly) in male Sprague-Dawley rats with initial body weight of approximately $310 \mathrm{~g}$. Four groups were studied: (a) vehicle $(n=12)$; (b) $\alpha$-MSH $(n=7)$; (c) SHU9119 (SHU; $n=5$ ); (d) Rats receiving SHU9119 and pair-fed to match the food intake of the $\alpha-\mathrm{MSH}$ group (SHU-PF; $n=5$ ). Caloric intake was monitored and adjusted in SHU-PF, and rats were studied 7 days thereafter. Fatfree mass was calculated from the whole body volume of distribution of water. BW, body weight; $\Delta$ BW, change in BW during the 1-week period; FM, whole body fat mass; FFM, fat-free mass. ${ }^{A} P<0.01$ vs. vehicle.

mately $6.0 \mathrm{ml}$ of fresh blood (obtained by heart puncture from a littermate of the test animal) and heparinized saline $(5 \mathrm{U} / \mathrm{ml})$ was infused.

Immunoprecipitation and immunoblotting. IRS-1 and IRS-2 protein content and tyrosine phosphorylation were determined in liver extracts. Liver samples were homogenized in RIPA buffer (50 mM Tris-HCl, $\mathrm{pH} 7.5$, $150 \mathrm{mM} \mathrm{NaCl}, 1 \mathrm{mM}$ EGTA, $1 \% \mathrm{NP}-40,0.25 \% \mathrm{Na}$ deoxycholate, $1 \mathrm{mM}$ PMSF, $1 \mu \mathrm{g} / \mathrm{ml}$ leupeptin, $1 \mu \mathrm{g} / \mathrm{ml}$ pepstatin, $1 \mu \mathrm{g} / \mathrm{ml}$ aprotinin, $1 \mathrm{mM} \mathrm{NaF}, 1 \mathrm{mM}$ orthovanadate). Specific Ab's (Upstate Biotechnology Inc., Lake Placid, New York, USA) were used to selectively immunoprecipitate IRS-1 and IRS-2 from aliquots of the supernatant containing $1 \mathrm{mg}$ of protein extracts, followed by precipitation with protein $A$ and protein $G$ beads (Pierce Chemical Co., Rockford, Illinois, USA). The immunoprecipitates were washed in ice-cold RIPA buffer and resuspended in $50 \mu \mathrm{l}$ of Laemmli buffer. The immunocomplexes were resolved on 8-10\% SDS-PAGE and transferred to nitrocellulose membranes. The blots were probed with either anti-IRS-1/IRS-2 Ab's or antiphosphotyrosine Ab's (Transduction Laboratories, Lexington, Kentucky, USA). The blots were washed twice and incubated with secondary Ab's (IgG linked to horseradish peroxidase) for 1.5 hour at room temperature with agitation. The immunocomplexes were visualized with enhanced chemiluminescence (Amersham Pharmacia Biotech, Piscataway, New Jersey, USA). Quantification was performed by densitometric scanning of the autoradiography.

Western blots of hypothalamic extracts were prepared essentially as described above, except that $60 \mu \mathrm{g}$ of protein were loaded in each lane. MCR4 immunoblots were performed using Ab's $(1 \mu \mathrm{g} / \mathrm{ml})$ purchased from Research Diagnostic (Flanders, New Jersey, USA). These $A b$ 's were raised in goat against a unique peptide corresponding to an amino acid sequence mapping near the carboxy terminus of rat MCR4. The specificity of these anti-MCR4 Ab's was tested by incubation in the presence of more than 100 -fold excess of peptide anti- gen. Blots were also probed with Ab's specific for MCR3 (Research Diagnostic) and insulin receptor beta subunit (Transduction Laboratories). Quantification was performed by densitometric scanning of at least four independent experiments.

All values are presented as the mean plus or minus SE. Comparisons among groups were made using repeated measures ANOVA where appropriate. Where $\mathrm{F}$ ratios were significant, further comparisons were made using Newman-Keuls test.

The study protocol was reviewed and approved by the Institutional Animal Care and Use Committee of the Albert Einstein College of Medicine.

\section{Results}

We aimed to generate bidirectional changes in the activity of hypothalamic melanocortin receptors in order to examine their impact on body composition and insulin action independent of changes in food intake. However, treatment with the potent antagonist of melanocortin receptors, SHU9119, increased cumulative food intake $(\sim 70 \%)$ (Table 1). This confirms the observation of Fan et al. (17), which showed that hypothalamic melanocortinergic neurons exert a tonic inhibitory effect on feeding behavior. To delineate whether this pathway plays a role in the regulation of insulin action and carbohydrate metabolism that is independent of its anorectic effects, we pair-fed rats treated with either vehicle, SHU9119, or $\alpha$-MSH. Plasma leptin (Table 2 ) and insulin levels were moderately increased $(\sim 2$ fold; $P<0.01)$ in rats receiving SHU9119 and fed ad libitum, while they were modestly decreased $(-30 \%)$ in rats receiving $\alpha-\mathrm{MSH}$, compared with vehicle. Finally, plasma corticosterone levels were similar in all experimental groups (data not shown).

Body composition was assessed by measuring the distribution space of body water with ${ }^{3} \mathrm{H}_{2} \mathrm{O}$ and by postmortem dissection of fat depots (13). The modest changes in body weight in rats treated with $\alpha-\mathrm{MSH}$ and SHU9119 were largely accounted for by changes in fat mass (Table 1). While fat-free mass was not markedly affected in any of the experimental groups, it tended to be lower in rats treated with SHU9119 and pair-fed (Table 1). Importantly, administration of $\alpha-\mathrm{MSH}$ caused a selective decrease ( $\sim 50 \% ; P<0.01$ vs. vehicle) in intra-abdominal adiposity (sum of omental, epididymal, and perirenal fat depots) (Figure 3 , a and b), while

\section{Table 2}

Plasma concentrations of glucose, insulin, leptin, and free fatty acids during the insulin clamp studies

$\begin{array}{lcccc} & \text { Vehicle } & \alpha-M S H & \text { SHU } & \text { SHU-PF } \\ \text { Glucose }(\mathrm{mM}) & 7.2 \pm 1.0 & 7.1 \pm 0.7 & 7.2 \pm 1.0 & 7.1 \pm 0.8 \\ \text { Insulin }(\mathrm{pM}) & 452 \pm 21 & 438 \pm 18 & 436 \pm 19 & 456 \pm 14 \\ \text { FFA }(\mathrm{mM}) & 0.44 \pm 0.08 & 0.42 \pm 0.10 & 0.45 \pm 0.09 & 0.39 \pm 0.08 \\ \text { Leptin }(\mathrm{ng} / \mathrm{ml}) & 1.2 \pm 0.2 & 0.8 \pm 0.1^{\mathrm{A}} & 2.3 \pm 0.1^{\mathrm{A}} & 1.1 \pm 0.3\end{array}$

The values represent steady-state levels obtained by averaging at least five plasma samples during the experimental period. FFA, free fatty acids. ${ }^{A} P<0.01$ vs. vehicle. 
a

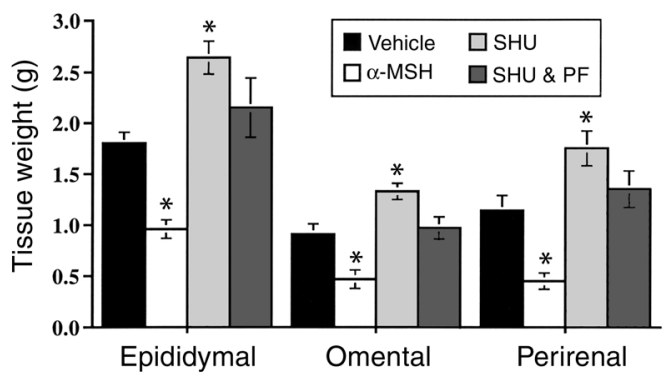

b

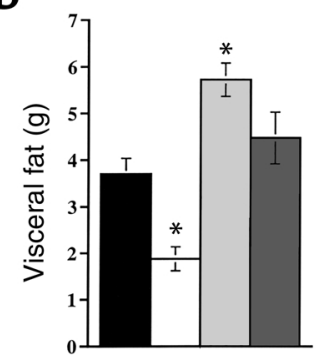

c

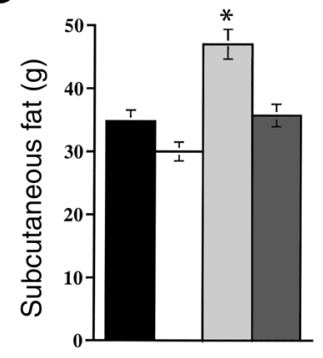

Figure 3

Effect of $\alpha$-MSH and SHU9119 administration on subcutaneous and visceral fat. (a) Weight of epidydimal, omental, and perirenal fat depots in the four experimental groups. (b) Total visceral fat mass represents the sum of omental, epididymal, and perirenal fat depots. (c) Subcutaneous fat mass was estimated by subtracting the total visceral fat from the whole body fat mass. Of note, administration of $\alpha-\mathrm{MSH}$ selectively decreased the size of all visceral fat depots compared with SHU-PF, despite similar food intake, weight changes, and subcutaneous fat mass. Values represent mean $\pm \mathrm{SE}$. ${ }^{*} P<0.01$ vs. vehicle.

subcutaneous fat mass was only increased in rats treated with SHU9119 when fed ad libitum (Figure 3c). These results indicate that $\alpha-\mathrm{MSH}$, in addition to its known effects on food intake and whole body fat mass, has selective actions on visceral or intra-abdominal adiposity. Since leptin exerts a similar action on fat distribution (13), it is likely that leptin activation of the melanocortin pathway (22) partly accounts for this effect. The relative distribution of body fat between subcutaneous and visceral sites determines the metabolic impact of adiposity and intra-abdominal adiposity is a risk factor for diabetes mellitus, arteriosclerosis, and mortality $(23,24)$. Our results suggest that the neural melanocortin pathway could play a major role in the regulation of fat distribution.

We next examined whether altering the activity of neural melanocortin receptors led to changes in the in vivo actions of insulin. Insulin action on carbohydrate metabolism can be assessed in conscious rats using a combination of the insulin $(3 \mathrm{mU} / \mathrm{kg} / \mathrm{min})$ clamp and tracer dilution techniques $(13,19,20)$. The plasma glucose, free fatty acids, and insulin concentrations during the insulin clamp studies were similar in the four groups (Table 2). During physiologic hyperinsulinemia (plasma insulin $\sim 450 \mathrm{pM}$ ), the rate of glucose infusion required to maintain the plasma glucose concentration at basal levels was markedly increased (56\%) by $\alpha-\mathrm{MSH}$ and

decreased (32\%) by SHU9119 (Figure 4a). Thus, modulation of central melanocortinergic neurons results in dramatic changes in insulin's ability to promote glucose disposal. The two major effects of insulin in vivo are to stimulate the uptake of glucose into peripheral tissues (mostly in skeletal muscle and adipose tissue) and to diminish the production of glucose by the liver. The rate of tissue glucose uptake in $\alpha$-MSH $(27.8 \pm 1.7$ $\mathrm{mg} / \mathrm{kg} / \mathrm{min})$ was $28 \%$ higher than in vehicle $(21.9 \pm 1.4$; $P<0.01$ ) (Figure $3 \mathrm{~b}$ ). Conversely, the rate of glucose uptake was decreased $(18 \%)$ in rats treated with SHU9119 (18.2 $\pm 1.4 \mathrm{mg} / \mathrm{kg} / \mathrm{min})$ compared with vehicle (Figure $4 \mathrm{~b}$ ). Similarly, $\alpha$-MSH markedly enhanced the action of insulin in inhibiting glucose production (Figure 3 c) $(1.3 \pm 0.6$ vs. $5.0 \pm 0.3 \mathrm{mg} / \mathrm{kg} / \mathrm{min} ; P<0.01)$. By contrast, SHU9119 diminished insulin's effect on glucose production $(8.2 \pm 0.9 \mathrm{mg} / \mathrm{kg} / \mathrm{min} ; P<0.01)$. The effect of insulin on glucose production can also be expressed as percentage of inhibition from basal levels (Figure 4d). Insulin inhibited glucose production by $90 \% \pm 5 \%$ in $\alpha-\mathrm{MSH}, 56 \% \pm 3 \%$ in vehicle, and $34 \% \pm 4 \%$ in SHU9119. During the insulin clamp studies, $54-61 \%$ of the changes in glucose metabolism (rate of glucose infusion) induced by either $\alpha$-MSH or SHU9119 were
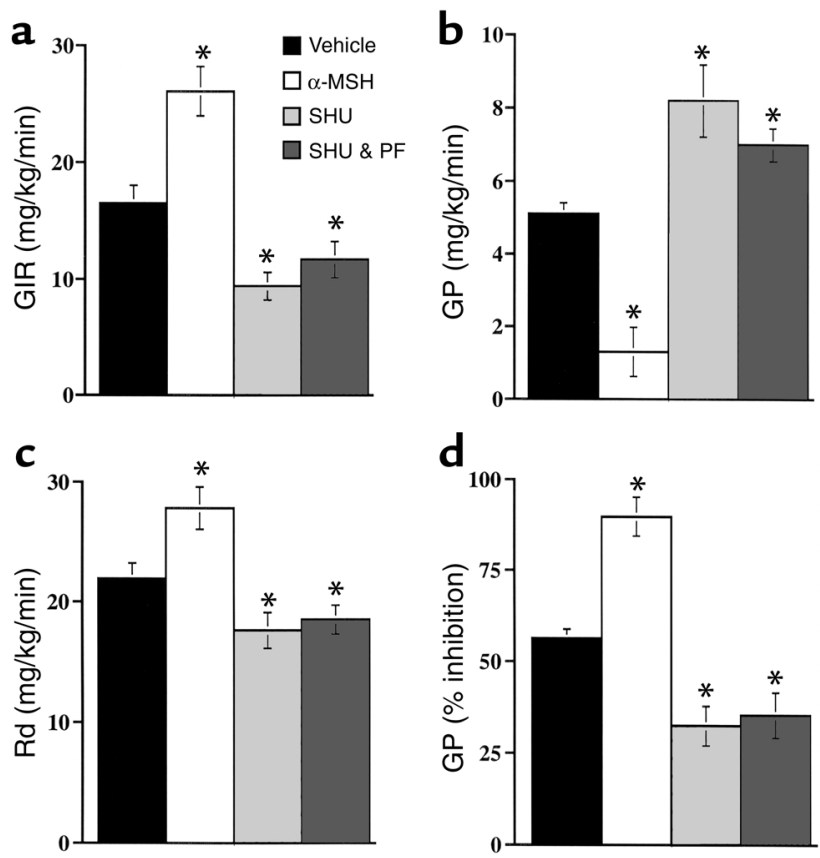

\section{Figure 4}

Role of the melanocortin pathway in the regulation of insulin action on peripheral glucose uptake and production. (a) During insulin clamp studies, the rate of glucose infusion was markedly increased by $\alpha-M S H$ and markedly decreased by SHU9119. (c) Insulin action on glucose uptake (Rd) was significantly enhanced by $\alpha-M S H$ and decreased by SHU9119. (b) During insulin clamp studies, the rate of glucose production (GP) was lower in rats treated with $\alpha-\mathrm{MSH}$ and higher in rats receiving SHU9119, compared with vehicle. (d) The inhibition of GP in response to physiological hyperinsulinemia was markedly increased by $\alpha-\mathrm{MSH}$ and markedly decreased by SHU9119. Values represent mean \pm SE. ${ }^{*} P<0.01$ vs. vehicle. GIR, glucose infusion rate. 
due to changes in glucose disposal and $39-46 \%$ were due to changes in glucose production. Thus, bidirectional modulation of central melanocortin receptors regulates both hepatic and peripheral insulin sensitivity. Since the metabolic effects of $\alpha$-MSH may be mediated by way of either type 3 or 4 melanocortin receptors, we next examined whether a selective decrease in hypothalamic MCR4 expression is sufficient to negate the effects of intracerebroventricular $\alpha-\mathrm{MSH}$ on fat distribution and insulin action. Intracerebroventricular delivery of ODN antisense has been shown previously to be effective in decreasing the expression of other central receptors (25). Here the intracerebroventricular infusion of ODN antisense to MCR4 generated a consistent decrease $(\sim 50 \%)$ in hypothalamic MCR4 protein as assessed by Western blot analysis (Figure 5, a-c). It should be noted that this decrease in whole hypothalamus is likely to underestimate the extent of the change in MCR4 protein in the hypothalamic nuclei surrounding the third cerebral ventricle. Intracerebroventricular administration of scrambled ODN did not alter the effect of $\alpha$-MSH on intraabdominal fat mass (decreased to $1.8 \pm 0.3 \mathrm{~g}$ ). However, the concomitant administration of ODN antisense to MCR4 blunted the effect of $\alpha$-MSH on intra-abdominal fat $(3.8 \pm 0.5 \mathrm{~g})$. Our results suggest that the neural melanocortin pathway regulates fat distribution largely by its stimulation of MCR4 in the hypothalamus.

We next examined whether decreasing the abundance of MCR4 in the hypothalamus alters the effect of the neural melanocortin pathway on in vivo insulin action. During physiologic hyperinsulinemia, the rate of tissue glucose uptake was increased in rats receiving $\alpha$-MSH and scrambled ODN (Figure 5d). Conversely, $\alpha-\mathrm{MSH}$ failed to increase the rate of glucose uptake in rats pretreated with ODN antisense to MCR4 (Figure 5c). Similarly, $\alpha-\mathrm{MSH}$ with scrambled ODN markedly enhanced the action of insulin in inhibiting glucose production (Figure 5e). By contrast, intracerebroventricular administration of ODN antisense to MCR4 negated the effect of $\alpha$-MSH on glucose production. Overall, these experiments support the notion that $\alpha-\mathrm{MSH}$ exerts its metabolic effects largely by hypothalamic MCR4.

To investigate potential mechanism(s) by which $\alpha-\mathrm{MSH}$ enhances hepatic insulin action, we measured the tyrosine phosphorylation of the two major substrates of the insulin receptor, IRS-1 and IRS-2, in the liver. In liver samples obtained at the completion of the insulin clamp studies, IRS- 1 and IRS- 2 proteins were present in similar amounts in all groups (Figure $6)$. However, tyrosine phosphorylation of IRS-1 (71\%; $P<0.01)$ and IRS-2 (98\%; $P<0.01)$ were markedly enhanced with $\alpha-\mathrm{MSH}$ compared with vehicle (Figure 6). Treatment with SHU9119 resulted in a modest decrease in IRS-1 and IRS-2 phosphorylation, which did not achieve statistical significance.

\section{Discussion}

Our results demonstrate that bidirectional modulation of central melanocortinergic neurons has a major impact on visceral adiposity and peripheral and hepatic insulin action. These effects involve the cluster of metabolic abnormalities that characterize the insulin-resistance syndrome in humans $(23,24)$. It is likely that the central melanocortin pathway regulates insulin action partly by its effect on visceral adiposity (26). Genetic models had suggested that impairment in central melanocortin receptor signaling not only results in obesity, but also in marked hyperinsulinemia and variable degrees of glucose intolerance $(15,27)$. For example, male MCR4 knockout mice (-/-) display hyperphagia, adult onset obesity and diabetes mellitus, and marked hyperinsulinemia. Importantly, a gene dosage effect has been demonstrated for this metabolic phenotype, with late onset hyperinsulinemia and glucose intolerance evident in the heterozygous (+/-) mice as well (15). Potent effects of the central melanocortin pathway on energy balance were also shown following short-term experiments in which specific agonist and antagonist of the MCR3/4
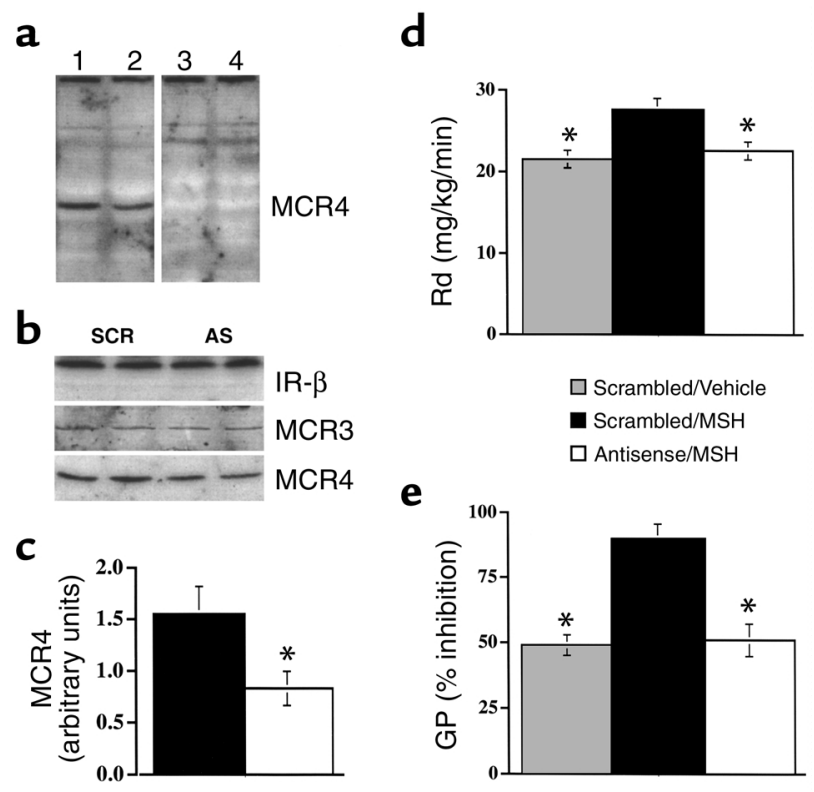

\section{Figure 5}

The MCR4 mediates the effect of $\alpha-M S H$ on hepatic insulin action. (a) Western blot of MCR4 in hypothalamus of rats receiving intracerebroventricular infusions of either scrambled ODN (SCR; lanes 1 and 3 ) or MCR4 antisense (AS; lanes 2 and 4 ) for 7 days. Representative blots are displayed. The identity of the 43-kDa band was confirmed by peptide competition. Addition of a specific MCR4 peptide eliminated this band (lanes 3 and 4), but failed to alter a nonspecific band. (b) Intracerebroventricular administration of MCR4 antisense decreased hypothalamic MCR4 protein, while insulin receptor IR- $\beta$ and MCR3 proteins were not affected. (c) Quantitation of hypothalamic Western blots: intracerebroventricular administration of MCR4 antisense led to a consistent, approximately $50 \%$ decrease in hypothalamic MCR4 protein. (d) During insulin clamp studies, the rate of glucose disappearance (Rd) was increased in rats treated with $\alpha-\mathrm{MSH}$ with scrambled ODN; however, $\alpha-M S H$ failed to increase $\mathrm{Rd}$ in rats receiving MCR4 antisense. (e) During insulin clamp studies, the rate of glucose production (GP) was lower in rats treated with $\alpha-\mathrm{MSH}$ with scrambled ODN; however, $\alpha$-MSH failed to decrease GP in rats receiving MCR4 antisense. Values represent mean $\pm \mathrm{SE}$. ${ }^{*} P<0.01$ vs. vehicle. 
a

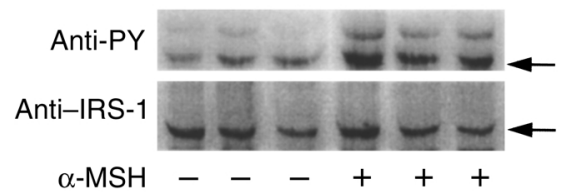

b

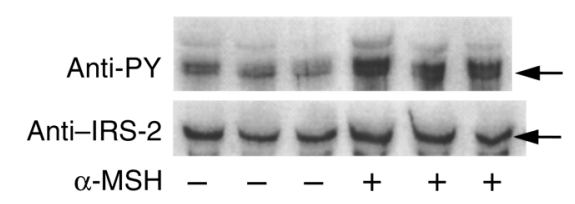

c

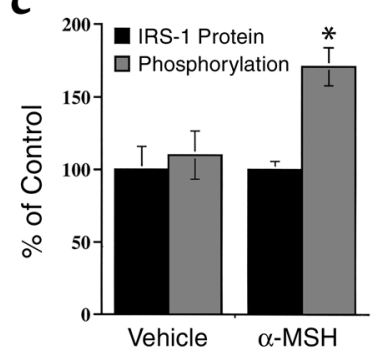

d

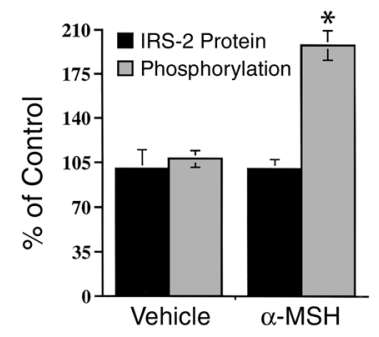

Figure 6

Effect of $\alpha-\mathrm{MSH}$ on hepatic insulin signaling. Liver samples were immunoprecipitated with anti-IRS-1 and anti-IRS-2 Ab's and then immunoblotted with anti-phosphotyrosine and anti-IRS1/2 Ab's. IRS-1 and IRS-2 proteins were not affected by treatment with $\alpha-\mathrm{MSH}$. By contrast, tyrosine phosphorylation of both insulin receptor substrates was markedly increased following $\alpha-\mathrm{MSH}$ treatment. Values represent mean $\pm \mathrm{SE}$. ${ }^{*} P<0.01$ vs. vehicle.

were given intracerebroventricularly (17). Indeed, here we show that hypothalamic melanocortinergic neurons exert a tonic modulatory effect not only on feeding behavior but also on fat distribution and insulin action. In fact, intracerebroventricular administration of the MCR3/4 antagonist SHU9119 for 7 days led to marked increases in food intake, rate of weight gain, and fat mass (Table 1) only in rats fed ad libitum. Conversely, SHU9119 led to increase in visceral adiposity and insulin resistance independent of its effect on feeding behavior.

In the present study, the intracerebroventricular administration of $\alpha-\mathrm{MSH}$ at the selected daily dose decreased fat mass and body weight gain in the absence of a significant decrease in average food intake. The lack of effect on cumulative food intake may be due to the relatively low dose selected in our studies and to the moderate decrease in food intake induced by surgery in the vehicle control rats. This finding suggests that modest and prolonged increases in $\alpha-\mathrm{MSH}$ enhance energy expenditure. We also observed a trend toward decreased body weight in the rats receiving SHU9119 with pair feeding. This may be relevant on two levels. First, it reinforce our major finding that bidirectional changes in the central melanocortin pathway markedly regulate peripheral and hepatic insulin action. In fact, a comparison of the two key groups, i.e., rats treated with $\alpha$-MSH and SHU9119/pair-fed, indicates that the opposite changes in insulin action in these groups are not due either to changes in food intake nor in body weight (both these parameters were well matched in these two groups). Second, treatment with SHU9119 led to an apparent increase in energy expenditure in the presence of pair feeding (Table 1). This may be due to the disruption of cross-talking among energy pathways, since MCR 3 may be involved in a feedback loop involving neurons in the hypothalamic arcuate nucleus. Thus, it is possible that pair feeding, by negating the marked effect of SHU9119 on food intake, unveiled the consequences of this interrupted communication on energy expenditure. It is also likely that alterations in the timing of feeding induced by pair feeding may also play a role. In fact, rats in this group are likely to rapidly consume their daily allotment of food and then enter a prolonged fasting state. This, in turn, may lead to increased motor activity (food-seeking behavior). Finally, the small differences in plasma leptin levels induced by $\alpha-\mathrm{MSH}$ and SHU9119 administration may also play a role. It should also be noted that weeklong intracerebroventricular administration of SHU9119 with pair feeding tended to preserve fat mass over lean mass (Table 1). While it was not a focus of the present study, this preliminary observation warrants further investigation.

Recent evidence places the neural MCR4 downstream of leptin and supports its role in mediating some of the effects of leptin on energy balance (14). In fact, activation of hypothalamic leptin receptors modulates MCR4 signaling by increasing the biosynthesis of the natural agonist, $\alpha-\mathrm{MSH}$, and by decreasing the expression of a natural antagonist, agouti-related protein (AGRP) (28). Importantly, antagonism of the neural MCR3/4 blunts the effects of leptin on food intake and body weight. Leptin has also been implicated in the regulation of insulin action and signaling $(9-11,13,29)$. Early studies had shown that the effects of leptin treatment on plasma glucose and insulin concentrations may precede its effects on body weight $(6,7)$. We had shown previously that insulin action is markedly enhanced in moderately obese rats treated with subcutaneous leptin compared with pair-fed rats (13). Recent work by Shimomura et al. (9) demonstrates dramatic effects of 12-day subcutaneous infusion of recombinant leptin on plasma insulin and glucose levels in fatless mice. It should be noted that acute intracerebroventricular or systemic administration of leptin to rats resulted in alterations in hepatic but not peripheral glucose fluxes (18). The latter acute metabolic effects were largely limited to a redistribution of hepatic glucose fluxes between gluconeogenesis and glycogenolysis (18). Preliminary results from our laboratory indicate that the acute administration of $\alpha-\mathrm{MSH}$ reproduces these acute effects of leptin on hepatic glucose fluxes (30). Thus, it is likely that the effects of both leptin and melanocortins on insulin action require longer stimulation of these pathways. Finally, Fan et al. (31) have recently demonstrated that activation of the hypothalamic melanocortin pathway acutely decreased plasma insulin concentrations in mice. Importantly, the 
same authors also point out that hyperinsulinemia and decreased glucose tolerance precede the onset of hyperphagia and weight gain in the MCR4 (knockout) mice. Of note, the marked improvement in insulin action observed with intracerebroventricular $\alpha-\mathrm{MSH}$ in the present study occurred despite a modest decline in plasma leptin concentrations. The downstream mechanism(s) by which leptin and melanocortins modulate fat distribution and hepatic and peripheral insulin action remains to be delineated. However, it is likely that these effects are at least in part linked to stimulation of autonomic outflow to the liver, adipose cells, and/or skeletal muscle $(18,26)$. Finally, it should be noted that since the week-long intracerebroventricular administration of agonists and antagonists is likely to reach multiple sites within the central nervous system, modulation of extrahypothalamic melanocortin receptors may also play a role in these metabolic effects.

Taken together, these results suggest that the effects of leptin on hepatic and peripheral insulin action are likely to involve the activation of central melanocortinergic neurons. Thus, one can hypothesize that changes in the activity of a common central pathway, which concomitantly regulates energy balance and insulin action, may account in part for the tight association of insulin resistance with weight gain and of increased insulin sensitivity with weight loss (Figure 1b). Diet-induced obesity and short-term overfeeding $(21,32,33)$ have been shown to result in diminished effects of leptin on regulation of energy balance. Further studies are warranted to determine whether the metabolic actions of leptin will also be affected and whether the melanocortin pathway will be "spared" in these models.

\section{Acknowledgments}

This work was supported by grants to L. Rossetti from the NIH (DK-48321 and DK-45024), the Diabetes Research \& Training Center (DK-20541), and the American Diabetes Association. Silvana Obici has been a recipient of a mentor-based postdoctoral fellowship from the American Diabetes Association.

1. McGarry, J.D. 1992. What if Minkowski had been ageusic? An alternative angle on diabetes. Science. 258:766-770.

2. Petersen, K.F., et al. 1998. 13C/31P NMR studies on the mechanism of insulin resistance in obesity. Diabetes. 47:381-386.

3. Zhang, Y., et al. 1994. Positional cloning of the mouse obese gene and its human homologue. Nature. 372:425-32.

4. Weigle, D.S., et al. 1995. Recombinant ob protein reduces feeding and body weight in the ob/ob mouse. J. Clin. Invest. 96:2065-2070.

5. Campfield, L., Smith, F.J., Guisez, Y., Devos, R., and Burn, P. 1995. Recombinant mouse $\mathrm{OB}$ protein: evidence for a peripheral signal linking adiposity and central neural networks. Science. 269:546-549.

6. Pelleymounter, M.A., et al. 1995. Effects of the obese gene product on body weight regulation in ob/ob mice. Science. 269:540-543.
7. Schwartz, M.W., et al. 1996. Specificity of leptin action on elevated blood glucose levels and hypothalamic neuropeptide Y gene expression in ob/ob mice. Diabetes. 45:531-535.

8. Kahn, B.B., and Rossetti, L. 1998. Type 2 diabetes: who is conducting the orchestra? Nat. Genet. 20:223-225.

9. Shimomura, I., Hammer, R.E., Ikemoto, S., Brown, M.S., and Goldstein, J.L. 1999. Leptin reverses insulin resistance and diabetes mellitus in mice with congenital lipodystrophy. Nature. 401:73-76.

10. Sivitz, W.I., Walsh, S.A., Morgan, D.A., Thomas, M.J., and Haynes, W.G. 1997. Effects of leptin on insulin sensitivity in normal rats. Endocrinology. 138:3395-3401.

11. Rossetti, L., et al. 1997. Short-term effects of leptin on hepatic gluconeogenesis and in vivo insulin action. J. Biol. Chem. 272:27758-27763.

12. Kamohara, S., Burcelin, R., Halaas, J.L., Friedman, J.M., and Charron, M.J. 1997. Acute stimulation of glucose metabolism in mice by leptin treatment. Nature. 389:374-377.

13. Barzilai, N., Massilon, D., Vuguin, P., Hawkins, M., and Rossetti, L. 1997. Leptin selectively decreases visceral adiposity and enhances insulin action. J. Clin. Invest. 100:3105-3110.

14. Seeley, R.J., et al. 1997. Melanocortin receptors in leptin effects. Nature. 390-349.

15. Huszar, D., et al. 1997. Targeted disruption of the melanocortin-4 receptor results in obesity in mice. Cell. 88:131-141.

16. Boston, B.A., Blaydon, K.M., Varnerin, J., and Cone, R.D. 1997. Independent and additive effects of central POMC and leptin pathways on murine obesity. Science. 278:1641-1644.

17. Fan, W., Boston, B.A., Kesterson, R.A., Hruby, V.J., and Cone, R.D. 1997. Role of melanocortinergic neurons in feeding and the agouti obesity syndrome. Nature. 385:165-168.

18. Liu, L., et al. 1998. Intracerebroventricular leptin regulates hepatic but not peripheral glucose fluxes. J. Biol. Chem. 273:31160-31167.

19. Rossetti, L., Smith, D., Shulman, G.I., Papachristou, D., and DeFronzo, R.A. 1987. Correction of hyperglycemia with phlorizin normalizes tissue sensitivity to insulin in diabetic rats. J. Clin. Invest. 79:1510-1515.

20. Wang, J., Liu, R., Hawkins, M., Barzilai, N., and Rossetti, L. 1998. A nutrient sensing pathway regulates leptin gene expression in muscle and fat. Nature. 393:684-688.

21. Wang, J., et al. 1999. The effect of leptin on Lep expression is tissue-specific and nutritionally regulated. Nat. Med. 5:895-899.

22. Schwartz, M.W., Seeley, R.J., Campfield, L.A., Burn, P., and Baskin, D.G. 1996. Identification of targets of leptin action in rat hypothalamus. J. Clin. Invest. 98:1101-1106.

23. Bjorntorp, P. 1991. Metabolic implications of body fat distribution. Diabetes Care. 14:1132-1143.

24. Kissebah, A.H. 1991. Insulin resistance in visceral obesity. Int. J. Obes. 15(Suppl. 2):109-115.

25. Whitesell, L., et al. 1993. Stability, clearance, and disposition of intraventricularly administered oligodeoxynucleotides: implications for therapeutic application within the central nervous system. Proc. Natl. Acad. Sci. USA. 90:4665-4672.

26. Barzilai, N., et al. 1999. Decreased visceral adiposity accounts for leptin effect on hepatic but not peripheral insulin action. Am.J. Physiol. 277:E291-E298.

27. Klebig, M.L., Wilkinson, J.E., Geisler, J.G., and Woychik, R.P. 1995. Ectopic expression of the agouti gene in transgenic mice causes obesity, features of type 2 diabetes, and yellow fur. Proc. Natl. Acad. Sci. USA. 92:4728-4732.

28. Haskell-Luevano, C., et al. 1999. Characterization of the neuroanatomical distribution of agouti-related protein immunoreactivity in the rhesus monkey and the rat. Endocrinology. 140:1408-1415.

29. Kellerer, M., et al. 1997. Leptin activates PI-3 kinase in C2C12 myotubes via janus kinase-2 (JAK-2) and insulin receptor substrate-2 (IRS-2) dependent pathways. Diabetologia. 40:1358-1362.

30. Liu, L., et al. 1999. Acute effects of a-Melanocyte-Stimulating Hormone on hepatic glucose fluxes. Diabetes. 48(Suppl. 1):A26.

31. Fan, W., et al. 2000. The central melanocortin system can directly regulate serum insulin levels. Endocrinology. 141:3072-3079.

32. Widdowson, P.S., Upton, R, Buckingham, R, Arch, J., and Williams, G. 1997. Inhibition of food response to intracerebroventricular injection of leptin is attenuated in rats with diet-induced obesity. Diabetes. 46:1782-1785.

33. Bjorbaek, C., Elmquist, J.K., Frantz, J.D., Shoelson, S.E., and Flier, J.S. 1998. Identification of SOCS-3 as a potential mediator of central leptin resistance. Mol. Cell. 1:619-625. 